\title{
Laboratory System for Quantum Cascade Lasers Researching
}

\author{
J. Mikolajczyk, W. Pichola, J. Wojtas*, M. Mamajek, M. Garlińska, A. Prokopiuk \\ AND Z. BIELECKI \\ Institute of Optoelectronics, Military University of Technology, S. Kaliskiego 2, 00-908 Warsaw, Poland
}

\begin{abstract}
The paper presents a prototype of a driving system designed to laboratory investigations of quantum cascade lasers. Significant requirements of these lasers operation, as well as a construction of the main components of the system were analyzed. During the performed investigations, a tuning range of both current pulses and temperature control operation were determined. Additionally the method of monitoring both current and voltage of the lasers was also described. As a summary, results of laboratory studies of the system with the use of commercial quantum cascade lasers were presented.
\end{abstract}

DOI: $10.12693 /$ APhysPolA.124.505

PACS: 85.60.Bt, 85.60.Jb, 42.55.Px, 42.72.Ai

\section{Introduction}

Quantum cascade (QC) lasers are used in many applications in the infrared and terahertz spectrum range [1-4]. They have such advantages as a compact design, high spectral power, and high repetition rate of emitted optical pulses [5]. Thanks to high precision spectral tuning to a specific wavelength, they are very useful in spectrometric measurements [6]. That is why QC lasers are very promising radiation sources in many civil and military fields [7-9]. In comparison with laser diodes, QC lasers (QCL) are driven with higher level of both current and voltage signals [10]. Thus QCLs' driver systems are more complicated and they are still under developing.

In the paper, integrated system MiQCL for QC lasers investigations was described. In contrast to commercially available drivers, the designed one is characterized by a wide range of tuning parameters (current and voltage, frequency and duty cycle). It is also equipped with temperature controller providing high accuracy of temperature stability.

\section{The driving system design}

The MiQCL device is an integrated system that provides QCLs operating point tuning, and next its stabilization. These kind of lasers generate high levels of heat, and their temperature has significant influence on their emitted spectrum and operation point [11]. The presented device makes it possible to control driving signals and temperature of the laser housing. For example, the MiQCL enables generation of current pulses with a maximum amplitude of $25 \mathrm{~A}$ and a voltage of $24 \mathrm{~V}$. Additionally, it is equipped with a thermoelectric modules controller, the power of which is about $120 \mathrm{~W}$. It allows

*corresponding author; e-mail: jwojtas@wat.edu.pl to temperature stabilize with accuracy of $0.01 \mathrm{~K}$. Therefore the MiQCL driver is very suitable in applications with QCLs.

The MiQCL driver can operate in pulsed mode and pulsed mode with DC biasing. The biasing signal can be generated by either internal bias unit or by external power supply with precise voltage stabilization. Applying an internal generator, both pulses duration time and their repetition rate can be set in the ranges from $200 \mathrm{~ns}$ to $50 \mu \mathrm{s}$ and from $10 \mathrm{~Hz}$ to $100 \mathrm{kHz}$, respectively. It is also possible to extend these ranges using external trigger signal (up to $10 \mathrm{MHz}$ at $20 \mathrm{~ns}$ duration time). For monitoring of the laser current and voltage signals, two special connectors were implemented. Thanks to this it is possible to monitor QCLs operation point. Therefore the developed device provides possibility to study lightcurrent-voltage characteristics (LIV) and to determine time parameters of a light pulses for different values of the laser temperature.

The MiQCL system was constructed using a laser cooling system (TEC controller, laser housing with thermoelectric module), pulsed current driver, DC biasing unit, microprocessor with a built-in programmable pulse generator, user panel and a data interface (Fig. 1).

The cooling system consists of a commercial TEC controller TC-36-25-RS232 type Tetech Co. and a modified laser housing LDM-4872 type, ILX Lightwave Co. $[12,13]$. The controller was applied to the laser temperature stabilization. When the selected parameters of the cooling system, e.g. setting temperature or maximal TEC current, exceed thresholds, the alarm signal is indicated. At the same time, all elements of the MiQCL system are disabled. Assuming a large amount of laser heat, it was necessary to apply a new high power Peltier module inside the laser housing.

The construction of the pulsed current driver is based on stabilized power supplies and a current switching unit. In the unit the PCO7120 driver module was used [14]. Analyses and investigation showed that its output current level is limited by time parameters of generated pulses (their frequency and duty cycle). Therefore some 
optimizations of the switching unit construction were necessary.

The DC biasing unit consists of a stabilized power supply $(24 \mathrm{~V}, 1 \mathrm{~A})$ and a transistors current source, the operating current range of which is $0-500 \mathrm{~mA}$. The driving current of the laser structure is the sum of the DC biasing current and the pulse current. All elements of the laser driver are functionally integrated using the microprocessor unit. The microprocessor also enables possibility to set internal generator parameters, and to communicate with a PC via USB. The photography of the MiQCL device and its scheme are presented in Fig. 1.
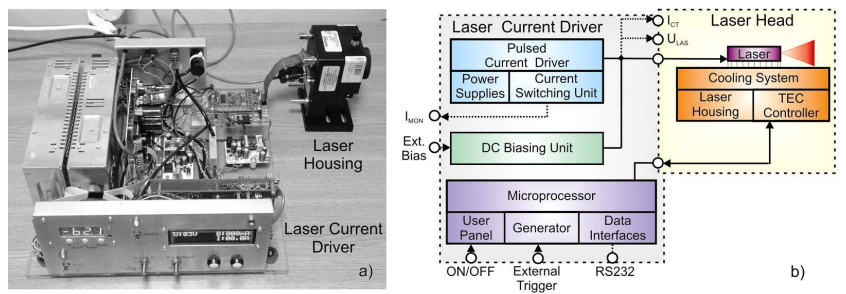

Fig. 1. The photography of MiQCL system (a) and its scheme (b).

\section{Preliminary research of QCL driving system}

Tests of the MiQCL device were performed using the non-inductive resistors. Their resistance value was similar to the level of the emitting laser resistance. The output current of the driver were determined using two data sources. Some values of $I_{\mathrm{CT}}$ were registered with the use of Tektronix CT-2 current probe or with the PCO7120 monitoring output. In addition, the output voltage was measured using two passive voltage probes. During the investigation, the main features of the MiQCL system were analyzed, e.g. a shape of generated pulses and $I-V$ characteristics for different values of load resistance $R_{\text {load }}$. The measurements were performed for two different connections between the MiQCL system and the load resistor. There were applied a copper-wires cabling and a low-impedance line. In both configurations, amplitudes of registered pulses had the same value. However, significant differences were noticed in pulse shape. They had influence on the pulses rise time $t_{\mathrm{r}}$ and fall time $t_{\mathrm{f}}$. For the low impedance line, values of $t_{\mathrm{r}}$ were in the range of 15-30 ns. In case of the cabling connection, the values of rise time were twice higher. Therefore, in the next measurements, the low impedance line was applied.

Next, the influence of the load resistance on pulses time parameters was determined. For voltage signal, the values of $t_{\mathrm{r}}$ do not exceed $5 \mathrm{~ns}$. However, in the case of the $I_{\mathrm{CT}}$ and $I_{\mathrm{MON}}$ the time rise values strongly depend on the load resistance value. Figure 2 shows some examples of voltage and currents oscillograms (a) and dependence of the rise time on the load resistance (b) as well. Significant oscillations are noticed in the case of voltage signals.

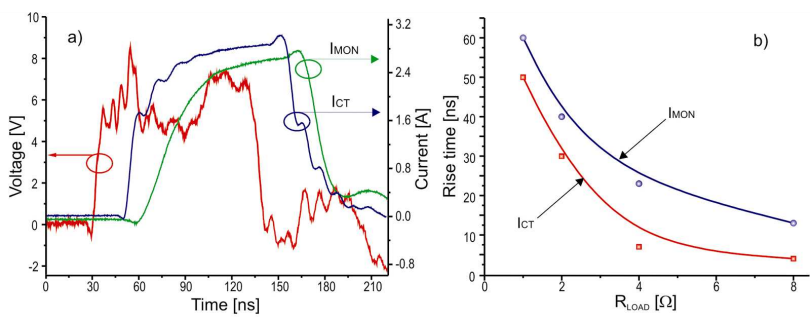

Fig. 2. The voltage and currents oscillograms (a) and the dependence of the rise time on the load resistance (b).
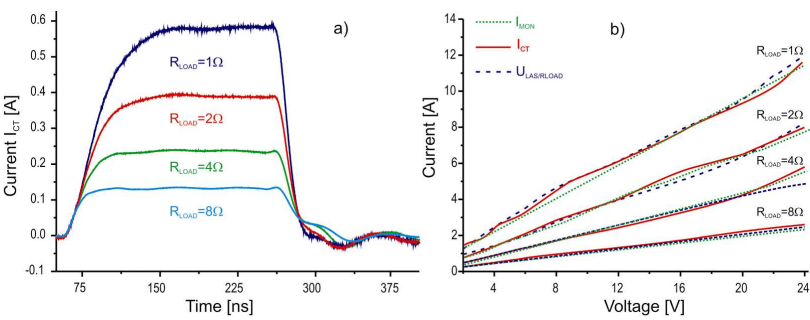

Fig. 3. The currents oscillograms for different values of load resistance (a), and the driver $I-V$ characteristics (b).

This effect results from e.g. the measurement method and the parameters of the applied voltage probes.

These measurements showed that increase in the load resistance causes decrease in the rise time value (Fig. 2b). This is due to a considerable influence of the load impedance on the driver features. Figure 3a shows the current oscillograms for different values of the load resistance. For these measurements, the CT-2 current probe was used. Based on these results, $I-V$ characteristics were also determined (Fig. $3 \mathrm{~b}$ ).

The $I-V$ characteristics show that dependence of driving current on voltage and the load resistance is linear. The insignificant differences between the measured values do not exceed the value of $5 \%$. Furthermore, the investigations have shown that it is possible to generate pulses with duration time of more than $10 \mu$ s and with repetition rate of above $10 \mathrm{MHz}$.

During the next step of the MiQCL researches, some parameters of the cooling system were determined. First, analyses of its cooperation with a commercial laser housing LDM-4872 were performed. During the experiment, a thermal camera v.50 (VIGO System S.A.) and an electric equivalent model of a $\mathrm{QC}$ laser with a resistance of about $2 \Omega$ were applied. The thermographs of the laser housing for temperature stabilization at $20^{\circ} \mathrm{C}$ and $0{ }^{\circ} \mathrm{C}$ are presented in Fig. 4. By analyzing the different temperature areas of the laser housing, it is noticed that the cooling system provides the effective cooling to a temperature of about $10^{\circ} \mathrm{C}$. Below this value, limitation of heat exchange (air cooling) was observed. To minimize this effect it would be necessary to apply additional external 


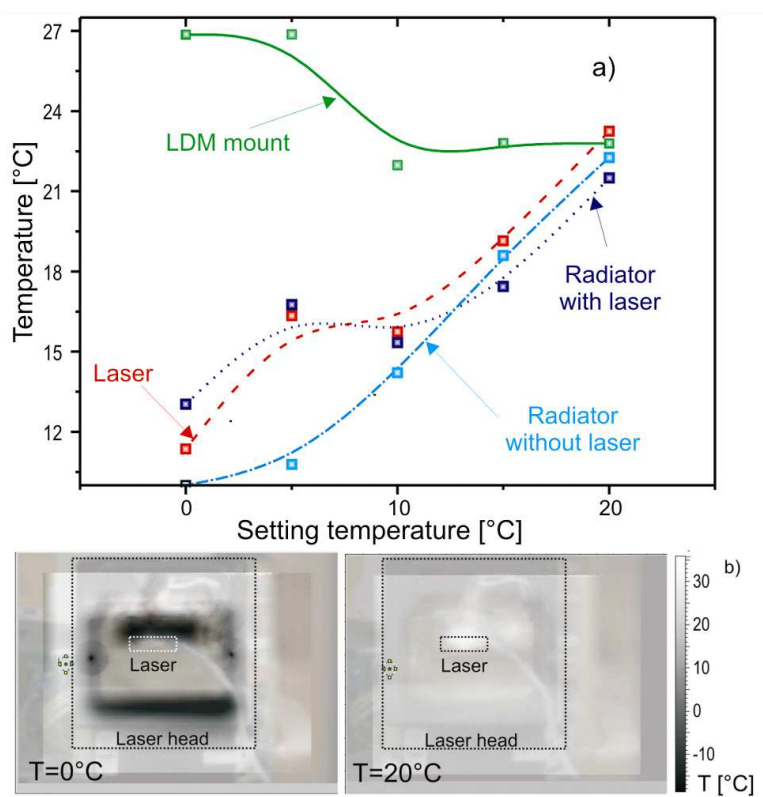

Fig. 4. A laser housing thermographs for temperature of $0{ }^{\circ} \mathrm{C}(\mathrm{a})$ and $20^{\circ} \mathrm{C}(\mathrm{b})$.

cooling e.g. with water.

\section{Researches of the MiQCL system with the use of quantum cascade lasers}

In the experiments, a QC laser from Alpes Lasers SA company was applied [15]. Moreover, additional water cooling was used, so the temperature range of laser operation was increased $\left(-50{ }^{\circ} \mathrm{C}-150^{\circ} \mathrm{C}\right)$. Peak power of the laser radiation was determined using a calibrated PVI-2TE-10.6 detection module VIGO System S.A. The LIV characteristics for different values of the laser temperatures are shown in Fig. 5. For the temperatures higher than $15^{\circ} \mathrm{C}$, QC laser stops emitting light. The results show that when temperature grows the laser threshold current increases, and the radiation power decreases. It should be noticed that when voltage reaches the value of between $9 \mathrm{~V}$ and $10 \mathrm{~V}$, power starts to reduce.

During the next studies, comparison of characteristics obtained using a commercial laser driver PCX1720 type and the MiQCL driver was carried out. It included the shape analysis of the laser radiation and the driving pulses (current and voltage). Some results for QC laser operation at $5{ }^{\circ} \mathrm{C}$ are presented in Fig. 6 .

Analyzing the shape of the driving pulses, some dynamic peaks are observed. The direct influence of the load impedance (laser and its cabling) on the current driver parameters is indicated. It is shown that the current connection technology of the laser structure and connection to the power supply is very important. For a low level of the driving current, two radiation peaks are observed. Moreover, shape of voltage signal at the detection module output is significantly different from the driving

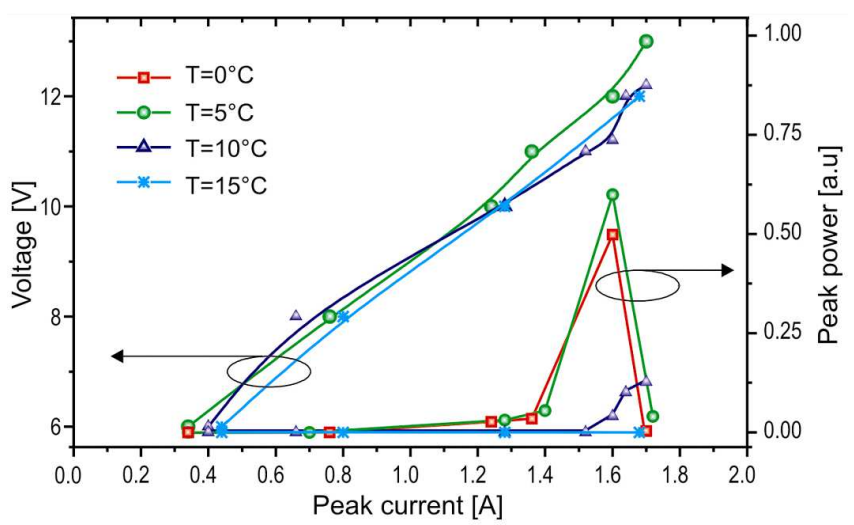

Fig. 5. Example of QCL LIV characteristics.

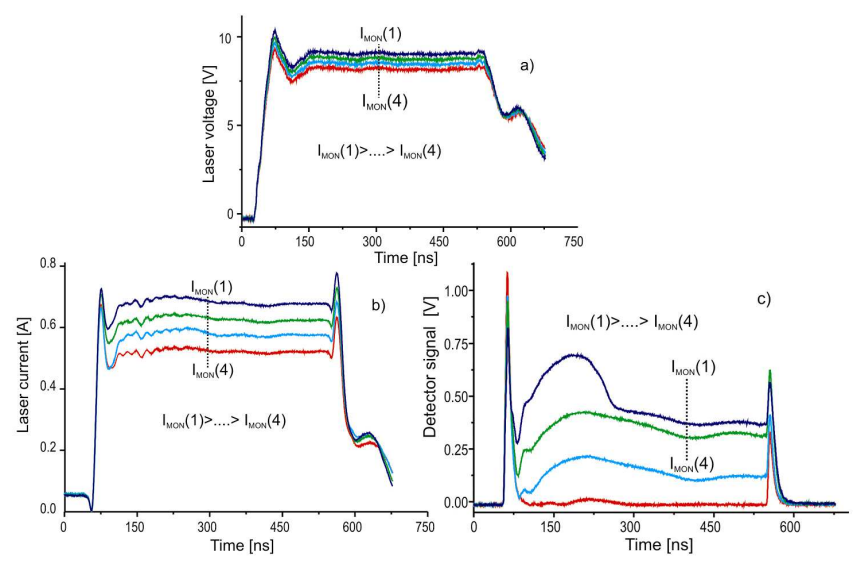

Fig. 6. QCL voltage (a) and current (b) oscillograms, voltage signal at the output of the detection module (c).

current pulse one. This effect may be caused by both laser construction and its electrical parameters.

Basing on registered voltage and current signals for different values of temperatures of QC laser, LIV characteristics were determined (Fig. 7). The average power of the optical signal at duty cycle of $0.05 \%$ was determined. The results were also compared with data obtained using PCX1720 driver.

There good data agreement can be observed. The results show also the influence of laser temperature on the threshold current and its radiation power.

\section{Conclusions}

The paper presents the specialized MiQCL driver to control and to stabilize the operating point of quantum cascade lasers. Its parameters provide the QC lasers testing in a wide range of both current and temperature values, as well as time parameters of driving pulses. It makes it possible to set a voltage in the range of $0-24 \mathrm{~V}$ and current of $0-25 \mathrm{~A}$. The MiQCL driver is equipped in a thermoelectric cooler controller, the power of which is about $120 \mathrm{~W}$. It allows to temperature stabilization with 


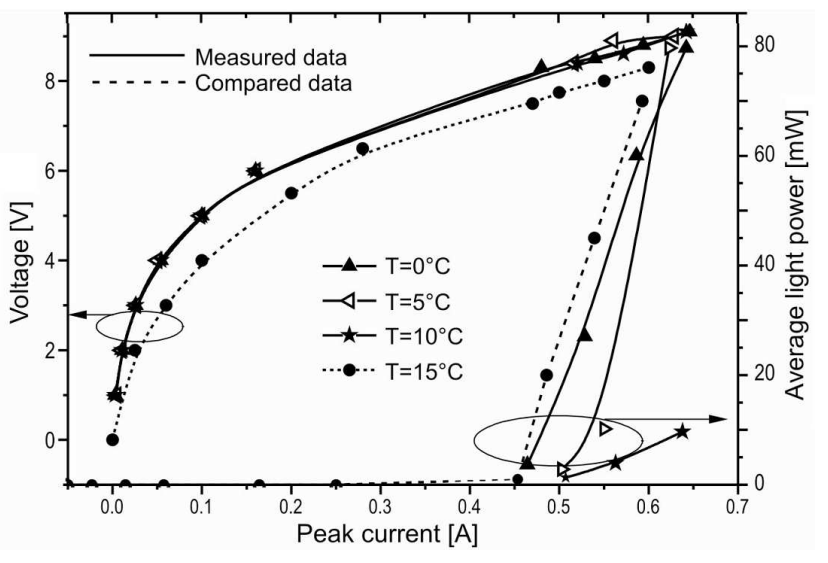

Fig. 7. Example of QCL LIV characteristics obtained during the MiQCL driver investigations.

accuracy of $0.01 \mathrm{~K}$. If water cooling is used, the temperature range is extended to value from $-50^{\circ} \mathrm{C}$ up to $150^{\circ} \mathrm{C}$. The investigation has shown that MiQCL driver allows to generate pulses with a duration time of $20 \mathrm{~ns}-10 \mu \mathrm{s}$ and with a repetition rate up to $10 \mathrm{MHz}$. In addition, the designed device has been equipped with some special signal connectors designed to the laser operation point monitoring, and also to apply an extra biasing current or an external synchronization signal. Furthermore, experimental results have shown that the MiQCL driver can be used for determining LIV characteristics. It could become a very important tool during the optimization and production processes of QC laser structures. Due to the driver system properties, it can be also used in researches of various applications such as laser communications and laser spectroscopy.

\section{Acknowledgments}

The authors would like to thank the Ministry of Science and Higher Education and The National Centre for Research and Development for their support in the field. The article was developed under research grants No. 179900 and O N515 216839.

\section{References}

[1] S.M. Cristescu, D. Marchenko, J. Mandon, K. Hebelstrup, G.W. Griffith, L.A.J. Mur, F.J.M. Harren, Appl. Phys. B 110, 203 (2013).

[2] R.F. Curl, F. Capasso, C. Gmachl, A.A. Kosterev, B. McManus, R. Lewicki, M. Pusharsky, G. Wysocki, F.K. Tittel, Chem. Phys. Lett. 487, 25 (2010).

[3] A. Rostami, H. Rasooli, H. Baghban, Terahertz Technol. Fundamentals Appl. Lecture Notes in Electrical Engineering 77, 191 (2011).

[4] R. Martini, E.A. Whittaker, Opt. Fiber Commun. Rep. 2, 393 (2008).

[5] J. Faist, T. Aellen, T. Gresch, M. Beck, M. Giovannini, NATO Sci. Peace Secur. Series B, Phys. Biophys., Eds. M. Ebrahim-Zadeh, I.T. Sorokina, 2008, p. 171.

[6] P. Malara, M.F. Witinski, F. Capasso, J.G. Anderson, P. De Natale, Appl. Phys. B 108, 353 (2012).

[7] J. Wojtas, Z. Bielecki, T. Stacewicz, J. Mikołajczyk, M. Nowakowski, Opto-Electr. Rev. 20, 26 (2012).

[8] C. Kumar, N. Patel, in: IEEE Photonics Society Summer Topical Meeting Series, 2011, Montreal 2011, p. 49.

[9] M. Gutowska, D. Pierścińska, M. Nowakowski, K. Pierściński, D. Szabra, J. Mikołajczyk, J. Wojtas, Z. Bielecki, Bull. Pol. Acad. Sci., Techn. Sci. 59, 419 (2011).

[10] K. Kosiel, A. Szerling, P. Karbownik, J. KubackaTraczyk, E. Pruszynska-Karbownik, A. Trajnerowicz, M. Bugajski, in: Int. Workshop Terahertz and Mid Infrared Radiation: Basic Research and Practical Applications, TERA-MIR 2009, Turunc-Marmaris 2009, p. 43.

[11] M. Wasiak, R.P. Sarzała, W. Nakwaski, Elektronika 1, 32 (2010) (in Polish).

[12] www.tetech.com/Temperature-Controllers/ TC-36- 25-RS232 .html

[13] www.newport.com/LDM-4872-Quantum-CascadeLaser-Diode-Mounting-Fix/1009659/1033/info.aspx .

[14] ixapps.ixys.com/DataSheet/pco-7120_data_ sheet.pdf .

[15] www.alpeslasers.com/lasers-on-stock/index. html\#lasers-on-stock/index_top. 